\title{
openheart Drivers and outcomes of variation in surgical versus transcatheter aortic valve replacement in Ontario, Canada: a population-based study
}

\author{
Rafael N Miranda (D) , ${ }^{1,2}$ Feng Qiu, ${ }^{3}$ Ragavie Manoragavan, ${ }^{4}$ Stephen Fremes, ${ }^{1,4}$ \\ Sandra Lauck, ${ }^{5}$ Louise Sun (1) , ${ }^{3,6}$ Christopher Tarola, ${ }^{4}$ Derrick Y Tam, ${ }^{4}$ \\ Mamas Mamas (D) , ${ }^{7}$ Harindra C Wijeysundera (D) ${ }^{1,4}$
}

\begin{abstract}
- Additional supplemental material is published online only. To view, please visit the journal online (http://dx.doi.org/10. 1136/openhrt-2021-001881).
\end{abstract}

To cite: Miranda RN, Qiu F, Manoragavan $\mathrm{R}$, et al. Drivers and outcomes of variation in surgical versus transcatheter aortic valve replacement in Ontario, Canada: a populationbased study. Open Heart 2022;9:e001881. doi:10.1136/ openhrt-2021-001881

Received 30 September 2021 Accepted 9 January 2022

Check for updates

(C) Author(s) (or their employer(s)) 2022. Re-use permitted under CC BY-NC. No commercial re-use. See rights and permissions. Published by BMJ.

For numbered affiliations see end of article.

Correspondence to Dr Harindra C Wijeysundera; Harindra.wijeysundera@ sunnybrook.ca

\section{ABSTRACT}

Objectives To understand the patient and hospital level drivers of the variation in surgical versus trascatheter aortic valve replacement (SAVR vs TAVR) for patients with aortic stenosis (AS) and to explore whether this variation translates into differences in clinical outcomes.

Background Adoption of TAVR has grown exponentially worldwide. Notwithstanding, a wide variation in TAVR rates has been seen within and between countries and in some jurisdictions AS is still primarily being managed by SAVR. Methods We conducted a population-based retrospective cohort study in Ontario, Canada, including individuals who received TAVR or SAVR between 2016 and 2020. We developed iterative hierarchical logistic regression models for the likelihood of receiving TAVR instead of SAVR examining sequentially patient characteristics, hospital factors and year of procedure, calculating the median ORs and variance partition coefficients for each. Using Cox proportional hazards models, we examined the relationship between TAVR/SAVR ratio on all-cause mortality and readmissions.

Results Annual procedures rates per million population increased from 171 to 201, mainly driven by the expansion of TAVR. TAVR/SAVR ratios differed substantially between hospitals, from 0.21 to 3.27. Neither patient nor hospital factors explained the between-hospital variation in AS treatment. The TAVR/SAVR ratio was significantly associated with clinical outcomes with high ratio hospitals having lower mortality and rehospitalisations.

Conclusions Despite the expansion of TAVR, dramatic variation exists that is not explained by patient or hospital factors. This variation was associated with differences in clinical outcomes, suggesting that further work is needed in understanding and addressing inequity of access.

\section{INTRODUCTION}

Aortic stenosis (AS) is the most common acquired valvular abnormality, with a prevalence of almost $10 \%$ in the elderly. ${ }^{1}$ It has been traditionally treated with surgical aortic valve replacement (SAVR), with a paradigm shift in the last decade after the introduction of a less invasive therapy, transcatheter aortic

\section{Key questions}

What is already known about this subject?

- Adoption of trascatheter aortic valve replacement (TAVR) has grown exponentially worldwide, but a wide variation in TAVR rates has been seen within and between countries and this variation can result into differences in clinical outcomes

What does this study add?

- TAVR/surgical aortic valve replacement (SAVR) ratios differed substantially between hospitals in Ontario Canada, and this variation was not explained by patient nor hospital factors. The TAVR/SAVR ratio was significantly associated with clinical outcomes, with higher ratio hospitals having lower mortality and readmissions

How might this impact on clinical practice?

- The finding of improved outcomes with a higher ratio of TAVR/SAVR merits further investigation. It may suggest that hospitals with a greater propensity to adopt new technologies also have greater emphasis on other quality improvement initiatives that translate to better outcomes.

valve replacement (TAVR). Currently, European and US guidelines recommend TAVR for patients at prohibitive risk for SAVR, and as an alternative for high, intermediate and low risk patients, based on the evaluation of the heart team and patient characteristics. ${ }^{2}$

With improvements in clinical experience, valve design and decreasing risk of procedural complications, ${ }^{3}$ the adoption of TAVR has grown exponentially worldwide. Notwithstanding, a wide variation in TAVR rates has been seen within and between countries. ${ }^{3-6}$ Previous research has explored potential causes for the varying rates of TAVR in different jurisdictions. Economic, specifically reimbursement and funding approaches, as well as infrastructure challenges have been 
cited as limiting factors in the deeper penetration of TAVR, with healthcare spending per capita correlated with higher procedural rates. ${ }^{36-9}$ In Canada, TAVR rates have been increasing over the last years, although at a slower rate when compared with other developed countries, ${ }^{10}$ with substantial variation between and within provinces. $^{71011}$ In Ontario, annual TAVR rates varied almost fourfold between different regions (31.5-119.5 TAVR per million population), ${ }^{5}$ with reports suggesting that AS is still primarily being managed by SAVR. ${ }^{12}$ There is a paucity of literature examining the underlying drivers for this variation in SAVR vs TAVR across hospitals.

Accordingly, to address this gap in knowledge, we conducted a population-based study in Ontario with two objectives: (1) to understand the patient and hospitallevel drivers of variation in TAVR and SAVR for AS, and (2) to explore whether clinical outcomes differed between hospitals based on TAVR/SAVR ratio. We hypothesise that the wide variation in SAVR vs TAVR rates in the province is not explained by measureable patient or hospital characteristics.

\section{METHODS}

\section{Study design and setting}

We conducted an observational retrospective cohort study using population based administrative data held at ICES, Ontario (previously known as the Institute for Clinical Evaluative Sciences). We adhered to the Strengthening the Reporting of Observational Studies in Epidemiology statement for reporting of observational studies. ${ }^{13}$

\section{Context}

The study was conducted in Ontario, which is Canada's largest province with a population of approximately 14.8 million. $^{14}$ All residents receive universal healthcare provided by a single third-party payer, the Ministry of Health. TAVR has been available in Ontario since 2012, and is currently funded for inoperable, high-risk, intermediate-risk and low-risk patients with AS. TAVR is currently available at all 11 hospitals in Ontario that provide SAVR.

\section{Data sources}

Our study used the CorHealth Ontario Clinical Registry as our primary data source. The database receives demographic, comorbidity and procedural data and its accuracy has been previously validated by means of retrospective chart review and comparisons with other databases. ${ }^{15}$ We linked the data using unique, encoded identifiers to administrative databases held at ICES. The Canadian Institute for Health Information Discharge Abstract Database provided data on acute hospitalisations, complications, as well as supplemented baseline comorbidity and procedural data. Validated ICES-derived databases were used to identify diabetes, ${ }^{16}{ }^{17}$ heart failure, ${ }^{18}$ hypertension, ${ }^{19}{ }^{20}$ chronic obstructive pulmonary disease, ${ }^{21}$ and dementia. ${ }^{22}$ Medical frailty was determined using the Hospital Frailty Risk Score. ${ }^{23}$ Mortality was ascertained via the Registered Persons Database as were additional demographic variables such as quintile of median neighbourhood income and rural residence.

\section{Study population}

We included all Ontario residents greater than 18 years of age who received TAVR or SAVR from 1 April 2016 to 31 March 2020. This time frame was selected to capture a contemporary AS population. Patients who received SAVR with or without coronary artery bypass graft $(\mathrm{CABG})$ were included only if the main diagnosis was predominantly AS (ICD-10 I350/I352), and those who underwent other concomitant cardiac procedures were excluded from this study. For patients who had repeated procedures during the period of the study, only the first procedure was included. Patients with a previous SAVR performed prior to 2016 were included in our primary cohort. Patients were excluded from the analysis if they had invalid birth, death dates or identifiers. The maximum follow-up date was 30 June 2020.

\section{Outcome measures}

For objective 1, the primary outcome was a completed TAVR or SAVR, treated as a dichotomous variable. We defined a TAVR/SAVR ratio for each hospital and categorised hospitals into tertiles of low, medium and high TAVR/SAVR ratio. We also treated TAVR/SAVR ratio as a continuous variable. For objective 2, our primary clinical outcomes were all-cause mortality and all-cause readmission until maximum follow-up.

\section{Statistical analysis}

We used a two-level hierarchical logistic regression model to investigate the between-hospital variability in the management of AS; we included hospital-specific random intercepts to account for clustering of patients by procedural hospital. We developed four models: (1) an unconditional/null model that contained the hospital-specific random effects only; models adjusted for; (2) patient characteristics; (3) patient characteristics and hospital factors; and finally (4) all variables, including the year of the procedure. For patient characteristics, we did not include the Charlson score, or ischaemic heart disease in the models, as it was colinear with other factors. We calculated two metrics of variation: the median OR (MOR) and variance partition coefficients (VPC) for each model. The MOR is defined as the median value of the OR for the outcome between individuals with identical covariates but randomly selected from different clusters (ie, hospitals), with clusters being ordered by TAVR/SAVR ratio. ${ }^{24}$ We calculated $95 \%$ crediblility intervals for the MOR. The VPC is the share of the total individual variance that is attributable to the cluster (ie, hospital) level; the higher the VPC, the more relevant is the influence of the cluster. ${ }^{24}$ As we add covariates to each model (null, vs 2, vs 3 , etc), if those covariates explained the variation between hospitals, we would expect the MOR and VPC to decrease. To provide a more comprehensive interpretation of the 
hospital-level effects, we calculated the $80 \%$ interval OR (IOR-80), which incorporates the magnitude of clusterlevel residual variations to the cluster-level covariates fixed effects. The IOR- 80 is the interval centred on the median of the distribution between the $10^{\text {th }}$ and $90^{\text {th }}$ percentiles of the values of the OR for each cluster-level covariate. If the interval includes the value one, the effect of the covariate is not as relevant when compared with the remaining cluster-level heterogeneity. ${ }^{25}$

To determine the consequences of variation in care, we developed Cox proportional hazards models with random intercepts (also known as frailty models) adjusted for patient characteristics, year of procedure and hospital factors, as well as wait-time and in-hospital complications, with the main factor being hospital TAVR/ SAVR ratio. For readmission, we developed a causespecific Cox proportional hazards model to account for the competing risk of death. In these models, the main exposure was the TAVR/SAVR ratio. We created two models: in the first, the TAVR/SAVR ratio was treated as a 3-level categorical variable; in the second, it was treated as continuous. We assessed if the TAVR/SAVR was nonlinear and if so, applied a restricted spline analysis. We repeated each of these models, excluded patients' admission status, hospital length of stay (LOS) and in-hospital periprocedural complications (bleeding, stroke, acute kidney injury and pacemaker/implantable cardioverterdefibrillator (ICD) insertion) to understand if these were the drivers of any outcome differences between hospitals in high, medium and low ratios of TAVR/SAVR.

We also repeated the models with an interaction term between the TAVR/SAVR ratios and receipt of either procedure to determine if the type of procedure (SAVR or TAVR) was an effect modifier of the clinical impact of variation.

\section{Sensitivity analysis}

We conducted a number of sensitivity analyses to determine the robustness of our models. We repeated our analyses, restricted to patients who underwent isolated SAVR and TAVR. We restricted our analyses to patients without a previous open-heart operation. We used SAS V.9.4 (SAS Institute). Statistical significance was considered to be two-sided $\mathrm{p} \leq 0.05$.

\section{RESULTS}

\section{Cohort}

We identified 5051 TAVRs performed in Ontario between 1 April 2016 and 31 March 2020; 24 of these were repeated procedures, yielding a total of 5027 unique TAVR patients. During the period of our study, of the 11855 SAVRs with or without CABG, 7436 had a primary diagnosis of AS. After excluding patients with concomitant multiple procedures, invalid codes and multiple procedures, 5577 patients who underwent SAVR were included in the analysis (figure 1).

The baseline characteristics of the patients are reported in table 1. TAVR patients were older (mean age 81.6 vs 70.4$)$, more likely female $(43.2 \%$ vs $32.3 \%)$ and had greater comorbidity burden, as well as prior procedures. As for the hospital factors, TAVR was more likely to be performed in hospitals with more acute care beds (mean 472.3 vs 440.6 ) but a smaller overall AS volume, based on total number of AS procedures (SAVR or TAVR) (mean 569.8 vs 581.3) over the study period. TAVR patients had greater wait times (median days 114 vs 57 days), while SAVR patients were more likely to undergo an urgent procedure, defined as a procedure occurring during an acute hospitalisation (14.7\% vs $18.4 \%)$.

Unadjusted outcomes are found on table 2. The only in-hospital complication that differed significantly between the two groups was pacemaker implantation, which was higher for TAVR (10.2\% vs $4.7 \%)$. TAVR patients had a shorter procedural LOS (median days 3 vs 7). There were no differences in crude 30 -day all-cause mortality or readmission. However, TAVR was associated
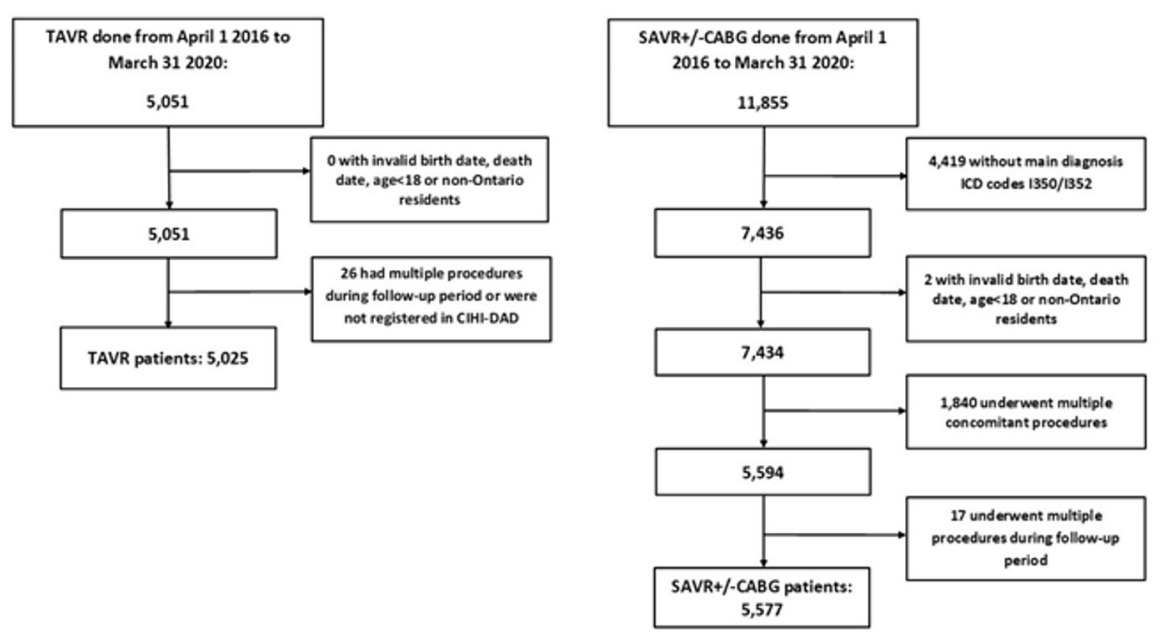

Figure 1 Cohort flow diagram. CABG, coronary artery bypass graft; CIHI-DAD, Canadian Institute for Health Information Discharge Abstract Database; ICD, implantable cardioverter-defibrillator; SAVR, surgical aortic valve replacement; TAVR, transcatheter aortic valve implantation. 
Table 1 Baseline patient characteristics by type of procedure for aortic stenosis (2016-2019)

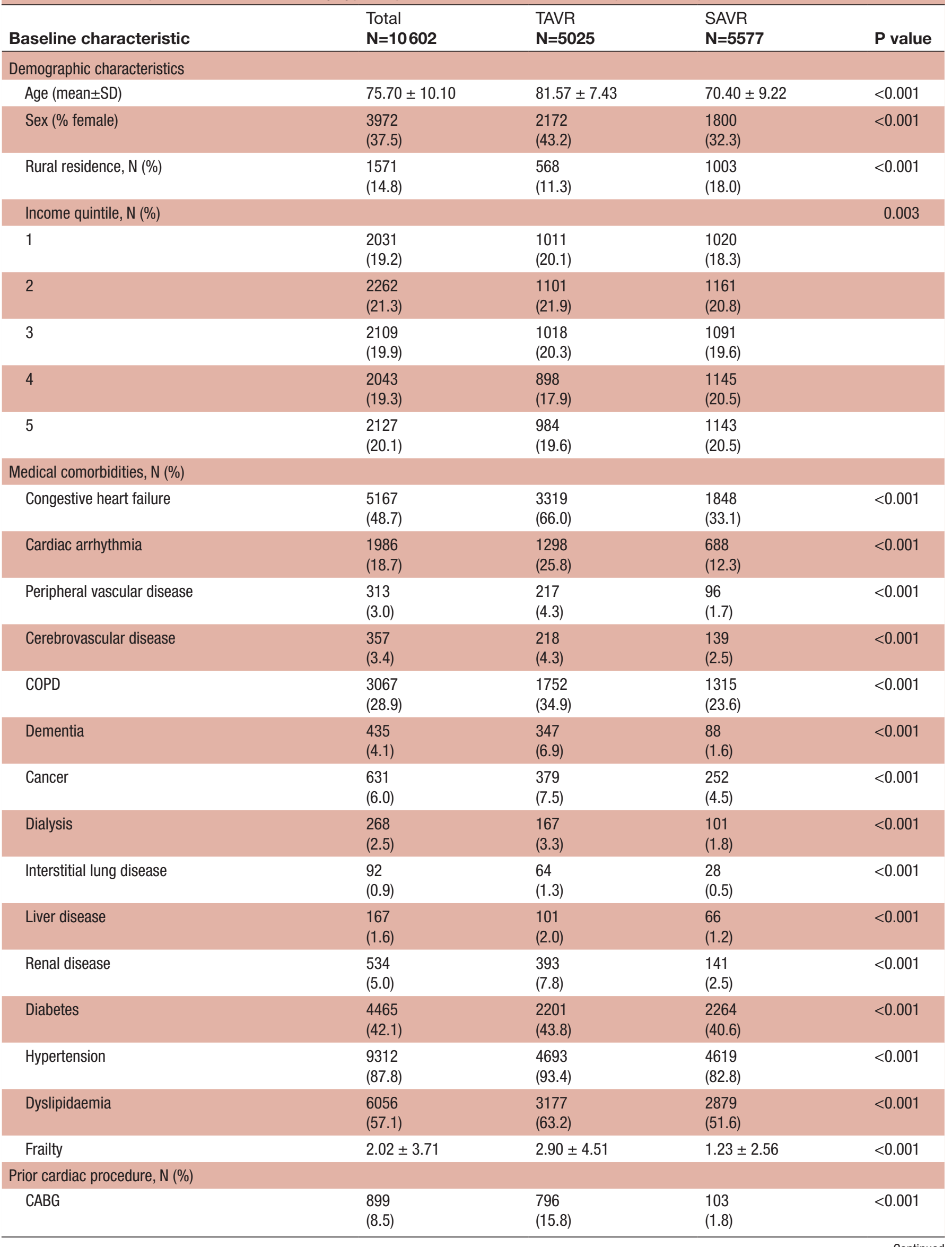




\begin{tabular}{|c|c|c|c|c|}
\hline Baseline characteristic & $\begin{array}{l}\text { Total } \\
\mathrm{N}=10602\end{array}$ & $\begin{array}{l}\text { TAVR } \\
\mathbf{N}=\mathbf{5 0 2 5}\end{array}$ & $\begin{array}{l}\text { SAVR } \\
\mathrm{N}=5577\end{array}$ & P value \\
\hline $\mathrm{PCl}$ & $\begin{array}{l}2347 \\
(22.1)\end{array}$ & $\begin{array}{l}1625 \\
(32.3)\end{array}$ & $\begin{array}{l}722 \\
(12.9)\end{array}$ & $<0.001$ \\
\hline Valve surgery & $\begin{array}{l}716 \\
(6.8)\end{array}$ & $\begin{array}{l}542 \\
(10.8)\end{array}$ & $\begin{array}{l}174 \\
(3.1)\end{array}$ & $<0.001$ \\
\hline Pacemaker (includes CRT-P) & $\begin{array}{l}591 \\
(5.6)\end{array}$ & $\begin{array}{l}473 \\
(9.4)\end{array}$ & $\begin{array}{l}118 \\
(2.1)\end{array}$ & $<0.001$ \\
\hline ICD (includes CRT-D) & $\begin{array}{l}99 \\
(0.9)\end{array}$ & $\begin{array}{l}77 \\
(1.5)\end{array}$ & $\begin{array}{l}22 \\
(0.4)\end{array}$ & $<0.001$ \\
\hline Prior stroke & $\begin{array}{l}191 \\
(1.8)\end{array}$ & $\begin{array}{l}115 \\
(2.3)\end{array}$ & $\begin{array}{l}76 \\
(1.4)\end{array}$ & $<0.001$ \\
\hline \multicolumn{5}{|l|}{ Hospital factors, N (\%) } \\
\hline Total acute care hospital beds (mean \pm SD) & $455.64 \pm 222.32$ & $472.33 \pm 213.79$ & $440.59 \pm 228.71$ & $<0.001$ \\
\hline Total no of procedures (mean \pm SD) & $581.31 \pm 180.81$ & $569.77 \pm 175.81$ & $575.84 \pm 178.54$ & $<0.001$ \\
\hline Teaching hospital & $\begin{array}{l}8303 \\
(78.3)\end{array}$ & $\begin{array}{l}4212 \\
(83.8)\end{array}$ & $\begin{array}{l}4091 \\
(73.4)\end{array}$ & $<0.001$ \\
\hline \multicolumn{5}{|l|}{ Status of procedure, $\mathrm{N}(\%)$} \\
\hline Urgent & $\begin{array}{l}1766 \\
(16.7)\end{array}$ & $\begin{array}{l}739 \\
(14.7)\end{array}$ & $\begin{array}{l}1027 \\
(18.4)\end{array}$ & $<0.001$ \\
\hline Elective & $\begin{array}{l}8836 \\
(83.3)\end{array}$ & $\begin{array}{l}4286 \\
(85.3)\end{array}$ & $\begin{array}{l}4550 \\
(81.6)\end{array}$ & $<0.001$ \\
\hline
\end{tabular}

CABG, coronary artery bypass graft; COPD, chronic obstructive pulmonary disease; CRT-D, cardiac resynchronisation therapy defibrillator; CRT-P, cardiac resynchronisation therapy pacemaker; ICD, implantable cardioverter-defibrillator; PCI, percutaneous coronary intervention; SAVR, surgical aortic valve replacement; TAVR, transcatheter aortic valve replacement; TIA, transient ischaemic attack.

with worse unadjusted outcomes at 90 days and maximum follow-up.

\section{Variation in procedures}

The overall number of procedures increased over the years, with 2393 being performed in 2016 and 2944 in 2019. This corresponds to an increase in procedures rates per million population from 171 to 201 (figure 2). TAVR rates increased from 61 to 122 , whereas SAVR rates decreased from 110 to 79 . TAVR/SAVR ratios differed substantially between hospitals, from 0.21 to 3.27 (figure 3). Mean TAVR/SAVR ratios for the entire province increased from 0.55 in 2016 to 1.53 in 2019 (online supplemental figure 1). Baseline patient and hospital characteristics by TAVR/SAVR ratio tertiles are found in online supplemental table S1.

\section{Drivers of AS care}

Figure 4 illustrates the drivers of receiving TAVR instead of SAVR. In general, increased comorbidity was associated with an increased likelihood of TAVR. For example, age (OR 1.22, 95\% CI 1.21 to 1.23), frailty score (OR $1.08,95 \%$ CI 1.06 to 1.1 ) and congestive heart failure (OR 2.14, 95\% CI 1.89 to 2.44). In addition, females were more likely to receive TAVR (OR 1.95, 95\% CI 1.72 to 2.2), as were patients who had previous procedures including PCI, CABG or pacemakers. As for the hospital factors, hospitals with a higher overall AS procedural volume over the entire study period were associated with a lower likelihood of TAVR (OR $0.66,95 \%$ CI 0.63 to 0.7 for every 100 procedures). There was a strong temporal effect, with TAVR more likely in the more recent years.

Patient variables, hospital factors and year of procedure all increased the MOR and VPC compared with the null model (online supplemental table S2). This indicates that neither patient, hospital factors nor year of procedure explained the between-hospital variation in receiving TAVR instead of SAVR. The IOR- 80 for the hospital factors in the full models were $0.15-8.44$ for acute care beds, 0.09-4.93 for number of procedures, and $0.57-31.65$ for teaching hospitals (online supplemental table S3). These wide intervals indicate that the residual variation between hospitals is substantial when compared with the hospital factors effects.

\section{Consequences of AS care}

When analysed as a categorical variable, The TAVR/SAVR ratio showed a significant association with all-cause readmissions (figure 5) with a gradient of improved outcomes with a higher ratio. Compared with high tertile hospitals, low and medium tertiles were associated with greater hazards of readmissions (HR 1.24, 95\% CI 1.08 to 1.43 and HR $1.13,95 \%$ CI 1.03 to 1.24 for low and medium tertiles, respectively). For all-cause mortality, the association was non-significant. 


\begin{tabular}{|c|c|c|c|c|}
\hline Baseline characteristic & $\begin{array}{l}\text { Total } \\
\mathrm{N}=10602 \\
\end{array}$ & $\begin{array}{l}\text { TAVR } \\
\mathbf{N}=\mathbf{5 0 2 5} \\
\end{array}$ & $\begin{array}{l}\text { SAVR } \\
\mathrm{N}=5577\end{array}$ & $P$ value \\
\hline Wait time (median days+IQR) & $\begin{array}{l}81 \\
(37-140)\end{array}$ & $\begin{array}{l}114 \\
(64-180)\end{array}$ & $\begin{array}{l}57 \\
(24-101)\end{array}$ & $<0.001$ \\
\hline \multicolumn{5}{|l|}{ Complications, N (\%) } \\
\hline Acute kidney injury & $\begin{array}{l}191 \\
(1.8)\end{array}$ & $\begin{array}{l}86 \\
(1.7)\end{array}$ & $\begin{array}{l}105 \\
(1.9)\end{array}$ & 0.508 \\
\hline Dialysis & $\begin{array}{l}226 \\
(2.1)\end{array}$ & $\begin{array}{l}111 \\
(2.2)\end{array}$ & $\begin{array}{l}115 \\
(2.1)\end{array}$ & 0.601 \\
\hline Bleeding & $\begin{array}{l}765 \\
(7.2)\end{array}$ & $\begin{array}{l}373 \\
(7.4)\end{array}$ & $\begin{array}{l}392 \\
(7.0)\end{array}$ & 0.434 \\
\hline Stroke/TIA & $\begin{array}{l}191 \\
(1.8)\end{array}$ & $\begin{array}{l}100 \\
(2.0)\end{array}$ & $\begin{array}{l}91 \\
(1.6)\end{array}$ & 0.166 \\
\hline Pacemaker during hospitalisation & $\begin{array}{l}774 \\
(7.3)\end{array}$ & $\begin{array}{l}513 \\
(10.2)\end{array}$ & $\begin{array}{l}261 \\
(4.7)\end{array}$ & $<0.001$ \\
\hline ICD & $\begin{array}{l}37 \\
(0.3)\end{array}$ & $\begin{array}{l}19 \\
(0.4)\end{array}$ & $\begin{array}{l}18 \\
(0.3)\end{array}$ & 0.629 \\
\hline CRT-P & $\begin{array}{l}39 \\
(0.4)\end{array}$ & $\begin{array}{l}23 \\
(0.5)\end{array}$ & $\begin{array}{l}16 \\
(0.3)\end{array}$ & 0.147 \\
\hline CRT-D & $\begin{array}{l}52 \\
(0.5)\end{array}$ & $\begin{array}{l}28 \\
(0.6)\end{array}$ & $\begin{array}{l}24 \\
(0.4)\end{array}$ & 0.35 \\
\hline \multicolumn{5}{|l|}{ Postoperative outcomes, N (\%) } \\
\hline Length of stay (median days+IQR) & $\begin{array}{l}6 \\
(3-10)\end{array}$ & $\begin{array}{l}3 \\
(1-7)\end{array}$ & $\begin{array}{l}7 \\
(5-11)\end{array}$ & $<0.001$ \\
\hline 30-day all-cause readmission & $\begin{array}{l}1084 \\
(10.2)\end{array}$ & $\begin{array}{l}539 \\
(10.7)\end{array}$ & $\begin{array}{l}545 \\
(9.8)\end{array}$ & 0.105 \\
\hline 90-day all-cause readmission & $\begin{array}{l}1831 \\
(17.3)\end{array}$ & $\begin{array}{l}972 \\
(19.3)\end{array}$ & $\begin{array}{l}859 \\
(15.4)\end{array}$ & $<0.001$ \\
\hline All-cause readmission to maximum follow-up & $\begin{array}{l}4553 \\
(42.9)\end{array}$ & $\begin{array}{l}2469 \\
(49.1)\end{array}$ & $\begin{array}{l}2084 \\
(37.4)\end{array}$ & $<0.001$ \\
\hline 30-day all-cause mortality & $\begin{array}{l}235 \\
(2.2)\end{array}$ & $\begin{array}{l}122 \\
(2.4)\end{array}$ & $\begin{array}{l}113 \\
(2.0)\end{array}$ & 0.161 \\
\hline 90-day all-cause mortality & $\begin{array}{l}369 \\
(3.5)\end{array}$ & $\begin{array}{l}219 \\
(4.4)\end{array}$ & $\begin{array}{l}150 \\
(2.7)\end{array}$ & $<0.001$ \\
\hline Overall mortality, to maximum follow-up & $\begin{array}{l}1425 \\
(13.4)\end{array}$ & $\begin{array}{l}929 \\
(18.5)\end{array}$ & $\begin{array}{l}496 \\
(8.9)\end{array}$ & $<0.001$ \\
\hline Follow-up from procedure* (median days+IQR) & $\begin{array}{l}657 \\
(333-1062)\end{array}$ & $\begin{array}{l}515 \\
(259-876)\end{array}$ & $\begin{array}{l}811 \\
(453-1177)\end{array}$ & $<0.001$ \\
\hline
\end{tabular}

*To death or end of study.

CRT-D, cardiac resynchronisation therapy defibrillator; CRT-P, cardiac resynchronisation therapy pacemaker; ICD, implantable cardioverterdefibrillator; SAVR, surgical aortic valve replacement; TAVR, transcatheter aortic valve replacement; TIA, transient ischemic attack.

When analysed as a continuous variable, we did not find any evidence of non-linearity in the TAVR/SAVR ratio. When modelled as a linear variable, findings were not consistent compared with the categorical models, and the association was significant for all-cause mortality, whereas for readmissions it was not (HR 0.87 with each unit increase in TAVR/ SAVR ratio, $95 \%$ CI 0.77 to 0.98 for mortality; HR 0.95 with each unit increase in TAVR/SAVR ratio, $95 \%$ CI 0.88 to 1.01 for readmission). The interaction term for procedures and TAVR/SAVR ratio was not statistically significant in any of our models. When we repeated our models, removing in-hospital complications and LOS, the results for all our models were consistent.

\section{Sensitivity analysis}

In the analyses restricting SAVR patients to isolated SAVR only and to patients with no previous open heart surgery, our overall results were qualitatively similar (online supplemental tables 4-11).

\section{DISCUSSION}

In this population-level study of care for AS patients, we found an overall increase in procedures for the management 


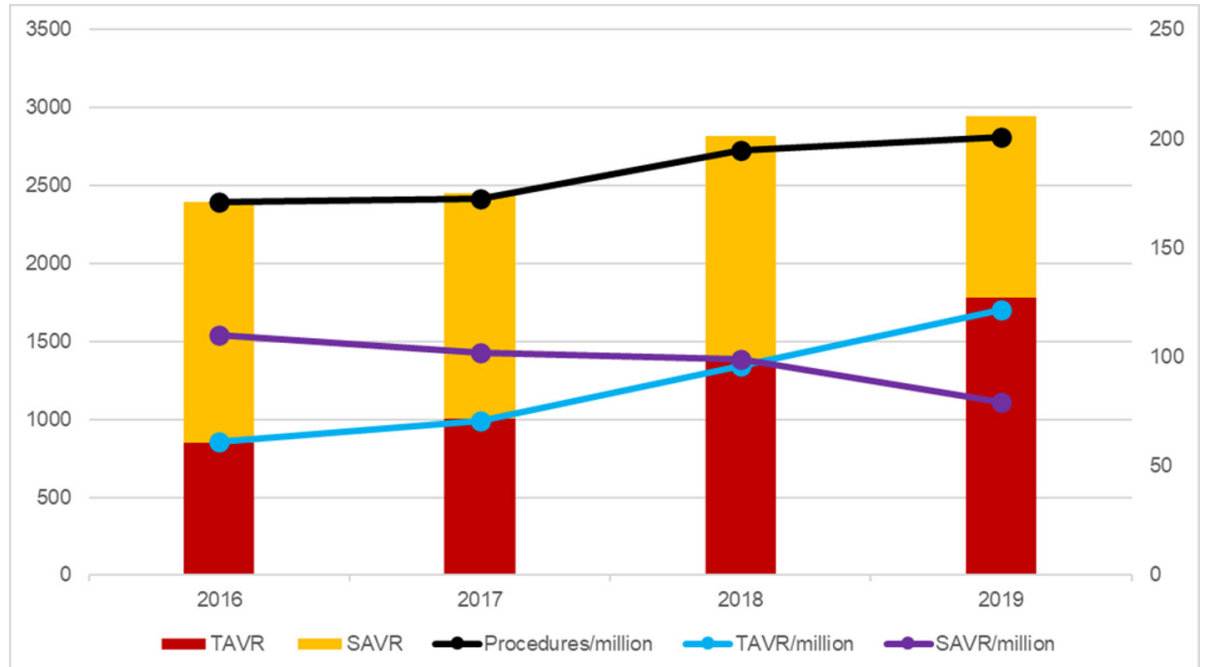

Figure 2 Number of procedures by hospital and fiscal year (2016-2019). SAVR, surgical aortic valve replacement; TAVR, trascatheter aortic valve replacement.

of AS, which was mainly driven by the expansion of TAVR, and a small decrease in SAVR. We found dramatic variations between hospitals in TAVR vs SAVR ratio. Although older and sicker patients underwent TAVR, the degree of variation was not accounted for by differences in patient characteristics, nor hospital characteristics. We found a gradient in clinical outcomes associated with this variation, with higher ratio hospitals associated with reductions in mortality and readmissions for AS patients, irrespective of TAVR or SAVR procedure type.

Previous work has shown variation in TAVR uptake within Ontario. ${ }^{5}$ Our study adds to this literature by confirming that patient differences are not the primary drivers of this variation. In other words, the same patient, visiting two different hospitals, may undergo a different procedure. In addition, it is not hospital factors either. Potential explanations for the variation may be reimbursement or funding strategies which are frequently cited as one of the main reasons for practice variation and late adoption of new technologies. ${ }^{46-8}$ Even in a single payer, universal healthcare model such as that in Canada, there are distinct funding lines for SAVR vs TAVR, and this may unintentionally incentivise practice variation. This may explain why larger valve centres have a lower propensity to TAVR as they would have a greater number of SAVR funded per year due to legacy funding, which in turn is an implicit disincentive for TAVR. Funding is a blunt instrument and thus there is a lag between clinical practice as recommended by guidelines and the ability of funding to keep pace. This should catalyse funding reform for adoption of more nimble approaches such as disease based, bundled funding models instead.

Other potential explanation for our findings is hospital culture. Broadly speaking, culture in this context can be conceptualised as whether the valve programme is more

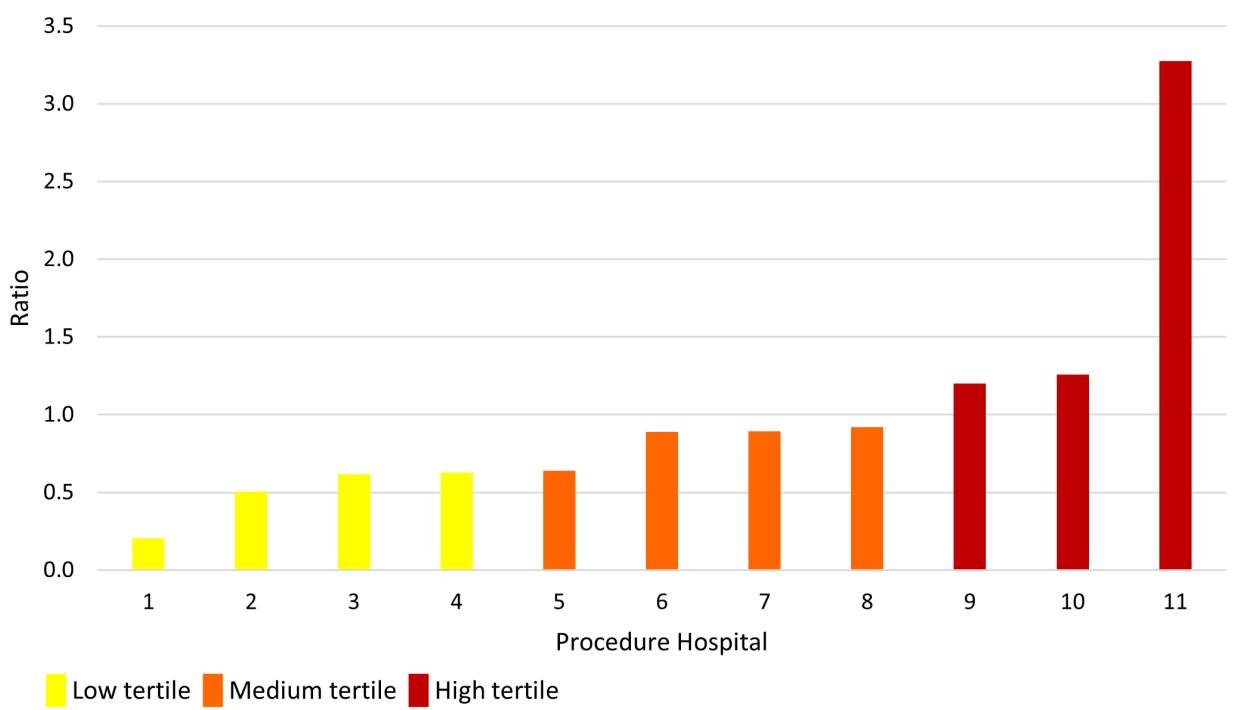

Figure 3 TAVR/SAVR ratios across hospitals in Ontario. SAVR, surgical aortic valve replacement; TAVR, trascatheter aortic valve replacement. 


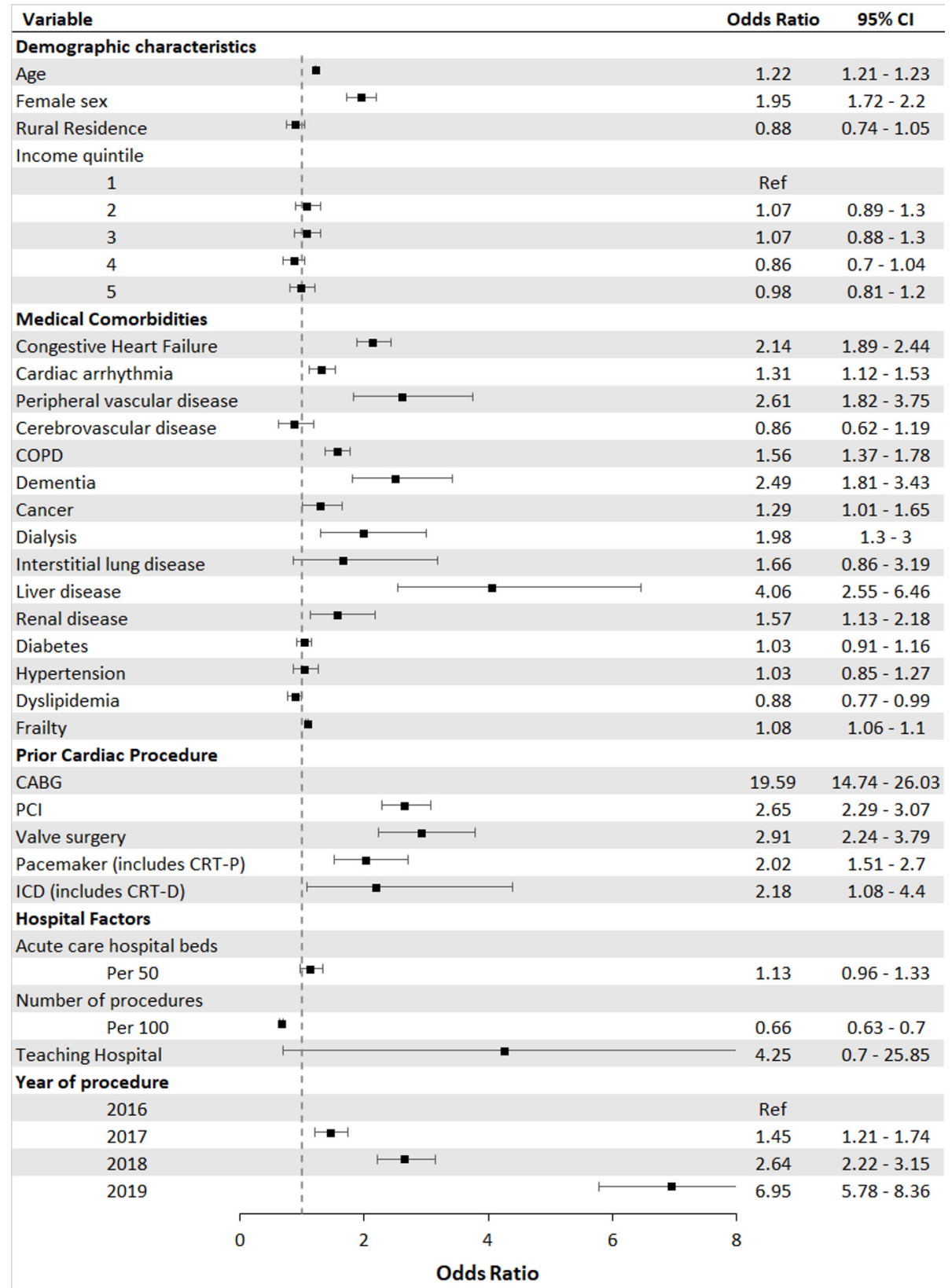

Figure 4 Factors associated with the likelihood of receiving TAVR instead of SAVR. Logistic model adjusting for patient, hospital factors and year of procedure. CABG, coronary artery bypass graft; COPD, chronic obstructive pulmonary disease; CRT-D, cardiac resynchronisation therapy defibrillator; CRT-P, CRT pacemaker; ICD, implantable cardioverter-defibrillator; $\mathrm{PCI}$, percutaneous coronary intervention; SAVR, surgical aortic valve replacement; TAVR, trascatheter aortic valve replacement.

surgical vs cardiology weighted and which group is the gate-keeper for AS referrals. The combined Heart Team is meant to mitigate this potential influence on treatment decisions. However, recent work from our group suggests a declining use of the Heart Team and that its use did not influence TAVR vs SAVR allocation. ${ }^{26}$

In so far as SAVR and TAVR variation, one could argue that such practice variations are not necessarily inappropriate or negative if there are no differences in clinical or economic outcomes between these treatments. Even in this setting of clinical and economic equivalence, there may be other consequences of unwarranted variation, such as patient satisfaction. Importantly, our work suggests that there may be a gradient in outcomes associated with variation in care, with higher ratio hospitals having improved outcomes. Interestingly, we had inconsistent findings when the TAVR/SAVR ratio was treated as a continuous or categorical variable in terms of benefit in mortality or readmission. In both cases, it was the the higher tertile hospitals that had improved clinical benefit. This was despite not finding any evidence of non-linearity. We hypothesise that the findings were simply from statistical power; that said, this association demands further investigation. To our knowledge, this is 


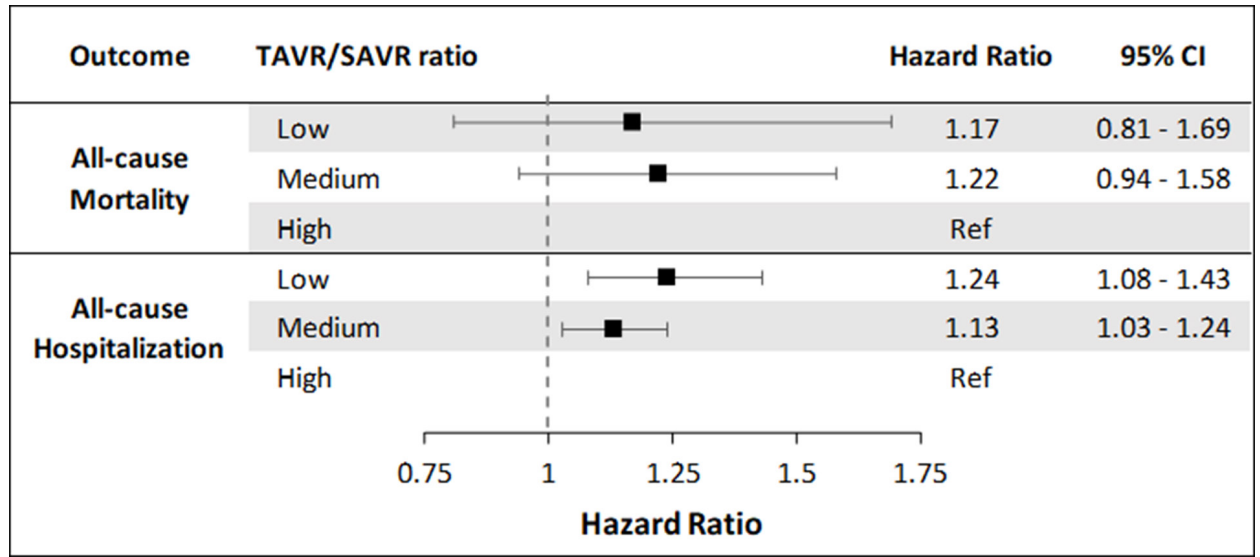

Figure 5 Association between TAVR/SAVR ratios and clinical outcomes. SAVR, surgical aortic valve replacement; TAVR, trascatheter aortic valve replacement.

the first study investigating variation in AS care and clinical outcomes.

Our study was not designed to understand the potential explanations for this difference. However, we can hypothesise as to various possibilities. First, we cannot rule out residual confounding as the cause. Second, this observation may be indicative of lower operator and institutional experience, which has been found to be associated with patient outcomes. ${ }^{27}$ The volume-outcome hypothesis does not explain why higher TAVR/SAVR ratios have improved outcomes even in SAVR patients. Finally, it may be that a hospital culture that is more ready to embrace new technologies (ie, higher TAVR/SAVR ratio), is also one that is more focused on quality improvement. Our results suggest that additional research is needed to confirm our findings and elucidate the underlying drivers.

\section{Limitations}

This study must be interpreted in the context of limitations that merit discussion. First, our study focused on variation of either SAVR or TAVR. Importantly, this requires that a patient with AS is diagnosed and referred for invasive therapies. As such, it is critical to acknowledge that our study may underestimate the degree of disparity, as there may be marked differences in the proportion of AS patients who are treated medically/conservatively. Given the substantial differences in outcomes between conservative therapy vs TAVR/SAVR, this disparity may translate to substantial variations in prognosis. Indeed, previous work in the USA suggests that there is geographic discordance between AS incidence and referral to therapy. ${ }^{29}$ It is an ongoing area of research in our group to understand if there are geographic, ethic and socioeconomic gradients in the referral for AVR therapies vs medical therapy in Ontario. Second, we were only able to include a few hospital-level factors due to data availability; however there may be additional hospital factors also relevant, such as number of trained professionals, diagnostic infrastructure, and availability of hospital facilities. ${ }^{58}$ Third, only mortality and readmission were measured as outcomes in the study, and variation in care may also have effects on additional outcomes such as functional capacity and quality of life. ${ }^{830}$ Finally, given the observation nature of the study, our findings should be considered hypothesis generating and not conclusive.

\section{CONCLUSIONS}

In conclusion, we observed an expansion in TAVR capacity across Ontario, although with a wide variation between hospitals. Ensuring patient driven, equitable access to both TAVR and SAVR remains a high priority in AS care.

\section{Author affiliations}

${ }^{1}$ Institute of Health Policy, Management and Evaluation, University of Toronto, Toronto, Ontario, Canada

${ }^{2}$ Toronto Health Economics and Technology Assessment (THETA) Collaborative, University Health Network, Toronto, Ontario, Canada

${ }^{3}$ Institute for Clinical Evaluative Sciences, Toronto, Ontario, Canada

${ }^{4}$ Schulich Heart Program, Sunnybrook Health Sciences Centre, Toronto, Ontario, Canada

${ }^{5}$ Centre for Heart Valve Innovation, Saint Paul's Hospital, Vancouver, British Columbia, Canada

${ }^{6}$ Division of Cardiac Anesthesiology, University of Ottawa Heart Institute, Ottawa, Ontario, Canada

${ }^{7}$ Keele Cardiovascular Research Group, School of Medicine, Keele University, Keele, UK

\section{Twitter Sandra Lauck @SandraLauck and Mamas Mamas @mmamas1973}

Acknowledgements The authors acknowledge that the clinical registry data used in this publication is from participating hospitals through CorHealth Ontario which serves as an advisory body to the Ontario Ministry of Health and Long-Term Care (MOHLTC), is funded by the MOHLTC, and is dedicated to improving the quality, efficiency, access and equity in the delivery of the continuum of adult cardiac, vascular and stroke services in Ontario, Canada.

Contributors All authors participated in the work and have reviewed and agree with the content of the article: RNM, FQ, RM, DYT and HCW participated in the conception and design of this study; RNM, FQ, RM and HCW conducted the data analysis; RNM drafted the manuscript; SF, SL, LS, CT, DYT and MM revised the manuscript for intellectual content; HCW is the guarantor of the study and approved the manuscript submission.

Funding This study was funded by a grant in aid from the Heart and Stroke Foundation of Canada (G-18-0020473). This study was supported by ICES, which is funded by an annual grant from the Ontario Ministry of Health (MOH) and the Ministry of Long-Term Care (MLTC). The analyses, conclusions, opinions and statements expressed herein are solely those of the authors and do not reflect 
those of the funding or data sources; no endorsement is intended or should be inferred. Parts of this material are based on data and/or information compiled and provided by Immigration, Refugees and Citizenship Canada (IRCC) current to 30 June 2020. However, the analyses, conclusions, opinions and statements expressed in the material are those of the authors, and not necessarily those of IRCC. Parts of this material are based on data and/or information compiled and provided by $\mathrm{ClHI}$. However, the analyses, conclusions, opinions and statements expressed in the material are those of the authors, and not necessarily those of $\mathrm{ClH}$. Parts of this material are based on data and information provided by Ontario Health $(\mathrm{OH})$. The opinions, results, view, and conclusions reported in this paper are those of the authors and do not necessarily reflect those of $\mathrm{OH}$. No endorsement by $\mathrm{OH}$ is intended or should be inferred. The authors acknowledge that the clinical registry data used in this publication is from participating hospitals through CorHealth Ontario, which serves as an advisory body to the $\mathrm{MOH}$, is funded by the $\mathrm{MOH}$, and is dedicated to improving the quality, efficiency, access and equity in the delivery of the continuum of adult cardiac, vascular and stroke services in Ontario, Canada. HCW is supported by a Tier 2 Canada Research Chair in Structural Heart Disease Policy and Outcomes. LS was named National New Investigator by the Heart and Stroke Foundation of Canada, and is supported by a Clinical Research Chair in Big Data and Cardiovascular Outcomes at the University of Ottawa.

Competing interests None declared.

Patient consent for publication Not applicable.

Ethics approval The use of data in this retrospective cohort study was authorised under section 45 of Ontario's Personal Health Information Protection Act, which does not require review by a Research Ethics Board.

Provenance and peer review Not commissioned; externally peer reviewed.

Data availability statement Data sets are not publicly available as per privacy legislation in Ontario, Canada.

Open access This is an open access article distributed in accordance with the Creative Commons Attribution Non Commercial (CC BY-NC 4.0) license, which permits others to distribute, remix, adapt, build upon this work non-commercially, and license their derivative works on different terms, provided the original work is properly cited, appropriate credit is given, any changes made indicated, and the use is non-commercial. See: http://creativecommons.org/licenses/by-nc/4.0/.

\section{ORCID iDs}

Rafael N Miranda http://orcid.org/0000-0003-2173-0021

Louise Sun http://orcid.org/0000-0003-3381-3115

Mamas Mamas http://orcid.org/0000-0001-9241-8890

Harindra C Wijeysundera http://orcid.org/0000-0001-8464-1080

\section{REFERENCES}

1 Leon MB, Smith CR, Mack M, et al. Transcatheter Aortic-Valve Implantation for Aortic Stenosis in Patients Who Cannot Undergo Surgery. N Engl J Med Overseas Ed 2010;363:1597-607. doi:10.1056/NEJMoa1008232

2 Cahill TJ, Terre JA, George I. Over 15 years: the advancement of transcatheter aortic valve replacement. Ann Cardiothorac Surg 2020;9:442-51. doi:10.21037/acs-2020-av-24

3 Cahill TJ, Chen M, Hayashida K, et al. Transcatheter aortic valve implantation: current status and future perspectives. Eur Heart $J$ 2018;39:2625-34.

4 Parma R, Dabrowski M, Ochała A, et al. The Polish interventional cardiology TAVI survey (PICTS): adoption and practice of transcatheter aortic valve implantation in Poland. Postepy Kardiol Interwencyjnej 2017;13:10-17.

5 Henning KA, Ravindran M, Qiu F, et al. Impact of procedural capacity on transcatheter aortic valve replacement wait times and outcomes: a study of regional variation in Ontario, Canada. Open Heart 2020;7:e001241.

6 Lutz M, Messika-Zeitoun D, Rudolph TK, et al. Differences in the presentation and management of patients with severe aortic stenosis in different European centres. Open Heart 2020;7:e001345.

7 McGuire C, Yip AM, MacLeod JB, et al. Regional differences in aortic valve replacements: Atlantic Canadian experience. Can J Surg 2018;61:99-104.

8 Merkel S, Eikermann M, Neugebauer EA, et al. The transcatheter aortic valve implementation (TAVI)--a qualitative approach to the implementation and diffusion of a minimally invasive surgical procedure. Implement Sci 2015;10:140.

9 Mylotte D, Osnabrugge RLJ, Windecker S, et al. Transcatheter aortic valve replacement in Europe: adoption trends and factors influencing device utilization. J Am Coll Cardiol 2013;62:210-9.

10 Asgar AW, Ouzounian M, Adams C, et al. 2019 Canadian cardiovascular Society position statement for transcatheter aortic valve implantation. Can J Cardiol 2019;35:1437-48.

11 Canadian Cardiovascular Society. National quality report: transcatheter aortic valve implantation. Ottawa, on, 2019.

12 Ontario Health (Quality). Transcatheter aortic valve implantation in patients with severe aortic valve stenosis at low surgical risk: a health technology assessment. Ontario Health Technology Assessment series 2020;20:1-148.

13 von Elm E, Altman DG, Egger M, et al. The strengthening the reporting of observational studies in epidemiology (STROBE) statement: guidelines for reporting observational studies. J Clin Epidemiol 2008;61:344-9.

14 Statistics Canada. Population estimates, quarterly. Available: https:// www150.statcan.gc.ca/t1/tbl1/en/tv.action?pid=1710000901 [Accessed 18 Apr 2021].

15 Wijeysundera HC, Qiu F, Koh M, et al. Comparison of outcomes of Balloon-Expandable versus Self-Expandable transcatheter heart valves for severe aortic stenosis. Am J Cardiol 2017;119:1094-9.

16 Guttmann A, Nakhla M, Henderson M, et al. Validation of a health administrative data algorithm for assessing the epidemiology of diabetes in Canadian children. Pediatr Diabetes 2010;11:122-8.

17 Hux JE, Ivis F, Flintoft V, et al. Diabetes in Ontario: determination of prevalence and incidence using a validated administrative data algorithm. Diabetes Care 2002;25:512-6.

18 Schultz SE, Rothwell DM, Chen Z, et al. Identifying cases of congestive heart failure from administrative data: a validation study using primary care patient records. Chronic Dis Inj Can 2013;33:160-6.

19 Tu K, Campbell NR, Chen Z-L, et al. Accuracy of administrative databases in identifying patients with hypertension. Open Med 2007;1:e18-26.

20 Tu K, Chen Z, Lipscombe LL, et al. Prevalence and incidence of hypertension from 1995 to 2005: a population-based study. Can Med Assoc J 2008;178:1429-35. doi:10.1503/cmaj.071283

21 Gershon AS, Wang C, Guan J, et al. Identifying individuals with physcian diagnosed COPD in health administrative databases. COPD 2009;6:388-94.

22 Jaakkimainen RL, Bronskill SE, Tierney MC, et al. Identification of physician-diagnosed Alzheimer's disease and related dementias in population-based administrative data: a validation study using family physicians' electronic medical records. J Alzheimers Dis 2016;54:337-49.

23 Gilbert T, Neuburger J, Kraindler J, et al. Development and validation of a hospital frailty risk score focusing on older people in acute care settings using electronic Hospital records: an observational study. Lancet 2018;391:1775-82. doi:10.1016/S0140-6736(18)30668-8

24 Austin PC, Wagner P, Merlo J. The median hazard ratio: a useful measure of variance and general contextual effects in multilevel survival analysis. Stat Med 2017;36:928-38.

25 Merlo J, Chaix B, Ohlsson $\mathrm{H}$, et al. A brief conceptual tutorial of multilevel analysis in social epidemiology: using measures of clustering in multilevel logistic regression to investigate contextual phenomena. J Epidemiol Community Health 2006;60:290-7.

26 Marcus G, Qiu F, Manoragavan R, et al. Temporal trends and drivers of heart team utilization in transcatheter aortic valve replacement: a Population-based study in Ontario, Canada. J Am Heart Assoc 2021;10:20741.

27 Wassef AWA, Rodes-Cabau J, Liu Y, et al. The Learning Curve and Annual Procedure Volume Standards for Optimum Outcomes of Transcatheter Aortic Valve Replacement: Findings From an International Registry. JACC Cardiovasc Interv 2018;11:1669-79. doi:10.1016/j.jcin.2018.06.044

$28 \mathrm{He} \mathrm{J}$, Zhang $\mathrm{Z}$, Wang $\mathrm{H}$, et al. The relation between volume and outcome of transcatheter and surgical aortic valve replacement: a systematic review and meta-analysis. Cardiovasc Ther 2020;2020:1-8

29 Vavalle JP, Phillips HR, Holleran SA, et al. Analysis of geographic variations in the diagnosis and treatment of patients with aortic stenosis in North Carolina. Am J Cardiol 2014;113:1874-8.

30 Bennell MC, Qiu F, Kingsbury KJ, et al. Determinants of variations in initial treatment strategies for stable ischemic heart disease. CMAJ 2015;187:E317-25. 